\title{
ACERCA DE LAS PAREJAS INCONGRUENTES Y LAS FIGURAS SIMÉTRICAS*
}

\author{
Carlos Álvarez \\ Facultad de Ciencias \\ Universidad Nacional Autónoma de México \\ alvarji@servidor.unam.mx
}

RESUMEN: Kant plantea el problema de las parejas incongruentes en 1768, posteriormente en 1770 y 1783 . Este problema, relacionado con su concepción acerca de la naturaleza del espacio, se vincula también con su idea sobre la naturaleza del conocimiento geométrico. Mi objetivo en este texto es analizar las observaciones de Kant sobre este punto - tres de las cuales son, a nuestro juicio, de suma relevancia - a partir de la geometría sólida euclidiana, la que constituye precisamente el marco teórico en el cual él pretende que ellas incidan.

PALABRAS CLAVE: congruencia, igualdad, ángulo sólido, conocimiento geométrico

SUMMARY: The question concerning the incongruent counterparts is raised by Kant in 1768, but it is also discussed later in 1770 and 1783. This question, which is related to his conception about the nature of space, is also closely related to his ideas about the nature of geometric knowledge. My aim in this paper is to analyze Kant's remarks on this topic - three of these remarks seem particularly relevant to us - in view of Euclidean solid geometry, which constitutes precisely the theoretic and scientific frame where he pretended they should fit.

KEY WORDS: congruence, equality, solid angle, geometric knowledge

\section{Introducción}

El caso de las parejas de cuerpos que son simétricos y que como tales son idénticos en todas sus partes pero que no son congruentes, i.e., que no es posible que uno de ellos cubra plenamente al otro, es analizado por Kant en tres ocasiones; la primera, en su pequeño opúsculo Del primer fundamento de la diferencia de las regiones del espacio (1768); dos años después

*Agradezco a un árbitro anónimo sus valiosas observaciones que me permitieron mejorar en forma y fondo la calidad de este trabajo. En cualquier caso es claro que la responsabilidad del contenido es sólo mía. 
en la De Mundi Sensibilis atque Intelligibilis Forma et Principiis, Dissertatio y, por último, en los Prolegómenos a toda metafísica futura (1783). ${ }^{1}$ Nos parece claro que este recurso al ejemplo de las parejas incongruentes ${ }^{2}$ deja ver hasta qué punto Kant toma conciencia de su importancia al considerar que más que una curiosidad, la explicación de este hecho le permite apuntalar su concepción acerca de la naturaleza del espacio.

En el periodo de 1768 a 1783, las ideas de Kant acerca del espacio evolucionan y pasan de una posición que busca probar la realidad propia del espacio absoluto, posición en la que parece aceptar plenamente la visión de Newton, a una posición característica del periodo crítico en el que abandona esta idea y en la cual el espacio es concebido como una intuición pura a partir de la cual es posible dar cuenta de las condiciones en las que la geometría es posible como ciencia pura. Pero ya sea que busque dar cuenta de la realidad del espacio al explorar filosóficamente, como lo declara en el opúsculo de 1768, el fundamento de aquello que Leibniz se proponía comprender matemáticamente a través del Analysis situs, o bien que pretenda establecer, como lo hará en 1783, el fundamento de una ciencia como lo es la geometría, la empresa de Kant requiere que la relación que guardan entre sí dos cuerpos simétricos y no congruentes sea plenamente explicada. Ello parece indicar que una clave para comprender la evolución de las concepciones kantianas sobre el espacio se encuentra en el modo en el que este problema es comprendido y asimilado. Sin embargo, mi objetivo en este texto no es analizar con detalle la evolución del pensamiento kantiano en torno de la naturaleza del espacio; más bien me propongo analizar los problemas geométricos a los que remite el ejemplo de las parejas incongruentes analizado por Kant. Con ello buscó comprender, desde esta perspectiva geométrica, la pertinencia de su visión ya que, como lo he dicho,

${ }^{1}$ En adelante me referiré a estas obras de Kant con las formas abreviadas de Regiones, Dissertatio y Prolegómenos, respectivamente.

${ }^{2}$ En su opúsculo sobre las Regiones del espacio, Kant introduce este término de la siguiente manera: "A un cuerpo que es perfectamente igual y similar a otro, pero que no se puede encerrar dentro de los mismos límites, lo llamo pareja incongruente." 
su concepción del espacio se encuentra íntimamente ligada al modo en el que él entiende este problema. ${ }^{3}$ Así, en la sección 2 comenzaré con una breve exposición de los tres momentos en que Kant recurre al ejemplo de las parejas incongruentes para que, una vez analizado con detalle el problema geométrico de la relación de simetría de los cuerpos en el espacio en la sección 3, podamos regresar a valorar la pertinencia y los límites de la comprensión de Kant acerca de este problema en la sección 4 .

La sección 2 no pretende ser una exégesis de la filosofía de Kant, ni siquiera en lo que se refiere a su teoría del espacio. Mi objetivo se limita a dar cuenta de dos aspectos: por un lado, el modo en el que Kant entiende el problema de estas parejas incongruentes y el papel específico que éste ocupa en relación con la naturaleza del conocimiento geométrico; junto con ello quiero también explicar de qué modo Kant llega a concebir la relación entre las parejas incongruentes y si acaso para él esta relación tiene la misma condición que la relación entre las parejas congruentes; es decir, si la relación geométrica de simetría - que se refiere a dos cuerpos idénticos en todas sus partes, pero en los que uno de ellos no cubre plenamente al otro- puede llegar a tener, y en qué condición, un valor similar al de la relación geométrica de congruencia, en la que además de la identidad en cada una de sus partes, una identidad global entre ambos permite que uno de ellos cubra plenamente al otro.

\section{De la interpretación filosófica al fundamento metafísico de una relación geométrica}

El interés de Kant en torno de la naturaleza del espacio ha tenido como telón de fondo una interpretación acerca de la naturaleza del conocimiento geométrico; por ello siempre es pertinente

${ }^{3}$ Este análisis se referirá exclusivamente a la ciencia geométrica y al conocimiento que del problema planteado por Kant se tiene hacia fines del siglo XVIII y principios del siglo XIX. La pertinencia de la visión de Kant en relación con la geometría y la topología de los siglos XIX y XX será analizada en una entrega posterior. 
preguntarse acerca de su conocimiento sobre la geometría, ya que si bien en general se ha dado por sentado que poseía un conocimiento sólido acerca de la ciencia newtoniana y la visión del espacio asociada a ella, en su texto de 1768, Regiones, él mismo señala que la reflexión que se impone para dar cuenta de la naturaleza absoluta - e independiente de toda materiadel espacio no debe partir de la ciencia del movimiento sino, de la geometría. A pesar de que nos parece claro su conocimiento de la obra filosófica de Descartes, no es posible asegurar que estuviera familiarizado con la Géométrie de 1637. Por otro lado, si a partir de este opúsculo de 1768 es posible asegurar que Kant conocía el texto de Euler sobre el espacio y el tiempo publicado en 1750, no existe indicio alguno de que conociera el resto de la obra geométrica de Euler, particularmente su trabajo sobre poliedros y cuerpo sólidos que fue publicado tiempo después. ${ }^{4}$ Sin embargo, podemos señalar con cierta seguridad, a partir de lo que él mismo deja ver, que Kant estaba familiarizado con algunos aspectos de la obra matemática de Leibniz, así como con la obra de Kästner. ${ }^{5}$ Si bien la influencia de ambos nos parece clara en el ensayo de 1768, la relación que Kant establece con el pensamiento de Leibniz merece atención especial. A pesar de que su objetivo central en este texto es mostrar la realidad del espacio absoluto, y de este modo hacer patente su filiación newtoniana, tomando así partido en un debate que enfrentaba desde hacía tiempo a los seguidores de Newton con los de Leibniz, Kant se limita sólo a hacer algunas alusiones indirectas al primero - a través del texto de Euler anteriormente citado-,

${ }^{4}$ El texto de Euler, Reflexions sur l'espace et le temps (1750) es citado por Kant también en su Ensayo para introducir el concepto de magnitud negativa en filosofía (1763). Los textos de Euler sobre los poliedros y los cuerpos sólidos, Elementa doctrince solidorum y Demonstratio nonnullarum insignium proprietatum, quibus solida hedris planis inclusa sunt praedita, datan de 1758.

${ }^{5}$ En el texto sobre las regiones del espacio, Kant hace una clara alusión a la Characterística Geometrica, así como a Die Analysis Geometrica propria und den Calculus situs betreffend y al Analysis situs de Leibniz. De Kästner conoce sin duda su Anfangsgründe der Arithmetik, Geometrie, ebenen un sphärischen Trigonometrie, und der Perspektiv, publicado en 1758, texto citado en la introducción de su ensayo sobre las magnitudes negativas. 
mientras que declara de manera explícita que la prueba que busca la obtendrá llevando adelante la valoración filosófica de lo que Leibniz intentó realizar matemáticamente bajo el proyecto del Analysis situs. Este interesante intento de usar a Leibniz contra Leibniz lo lleva a tomar una prudente distancia respecto de Euler, a pesar de compartir su filiación newtoniana. Mientras que los argumentos presentados por Euler a favor del espacio absoluto descansan sobre el hecho de que sin la hipótesis de su existencia no serían posibles los principios de la ciencia del movimiento, ciencia que él considera tan sólidamente fundada que no es posible dudar de su verdad, Kant toma como punto de partida la idea de Leibniz para constituir a partir de ella un argumento geométrico capaz de mostrar la existencia del espacio absoluto:

La prueba que yo busco aquí debe poner en manos, no sólo de los mecánicos como intentaba el señor Euler, sino incluso de los geómetras, una razón convincente para que puedan afirmar la realidad de su espacio absoluto con la evidencia a que están acostumbrados. ${ }^{6}$

Para Kant, el análisis de la posición o situación de las partes componentes de los cuerpos y las figuras, tal y como éstas se presentan y ordenan entre sí —análisis que deja de lado toda consideración respecto de su magnitud-, no puede por sí mismo dar cuenta de la forma corpórea y para ello requiere la existencia del espacio absoluto: el orden en el que estas partes se presentan sólo puede determinarse con el establecimiento de un sistema de referencia y de un observador que pueda localizarlas en relación con este sistema, pero ambos adquieren este carácter sólo a partir del espacio absoluto y real en el que se encuentran. Sin embargo, esta dependencia de la forma respecto del espacio absoluto deviene a su vez una prueba de la naturaleza absoluta del espacio al tomar en cuenta la existencia de las parejas incongruentes, ya que si el espacio sólo consistiese en la relación de situación de los objetos que coexisten unos con

${ }^{6}$ Regiones, $\S \mathrm{I}$. 
otros, como lo asegura el propio Leibniz, entonces la existencia de uno de dos cuerpos simétricos agotaría todo el espacio que a partir de él se define, y haría imposible la existencia del otro cuerpo, toda vez que, de acuerdo con esta concepción, ambos determinarían el mismo espacio y deberían, por lo tanto, ser congruentes.

Por ello, Kant asegura que el análisis de la situación es posible sólo si el espacio absoluto existe, y entonces toda teoría que a ello se dedique debe asumir su existencia como condición de posibilidad. De este modo, el ejemplo de los cuerpos incongruentes sirve como apoyo para extraer la prueba de la existencia del espacio absoluto a partir de su indagación filosófica acerca del primer fundamento de la teoría matemática leibniciana del Analysis situs. ${ }^{7}$

La discusión sobre la naturaleza del espacio y los sólidos simétricos y no congruentes no se agota con el texto de 1768; dos años después ambos temas son retomados en la Dissertatio. Este texto, considerado un punto de inflexión en la obra de Kant, marca el fin del periodo precrítico y anuncia la apertura del horizonte problemático característico de la filosofía crítica. ${ }^{8}$ La investigación sobre los principios del mundo sensible y del mundo inteligible, tema de esta Dissertatio, proporciona, mediante el establecimiento de los principios formales de ambos, el fundamento de lo que será más adelante la Estética trascendental.

La sensibilidad es concebida como una capacidad receptiva del sujeto ante la presencia de los objetos, mientras que la inteligencia se concibe como la facultad del sujeto de representarse aquello que por su naturaleza no se presenta a los sentidos. De este modo, el conocimiento sometido a las leyes de la sensibilidad es un conocimiento sensible, mientras que el sometido a la inteligencia es un conocimiento intelectual o racional. Conforme

${ }^{7}$ Regiones, §I.

${ }^{8}$ De acuerdo con H.J. de Vleeschauwer (1934), Kant señalaba (1797) la pertinencia de dejar fuera de la edición de sus obras todas las escritas antes de la Dissertatio y a tomar precisamente esta obra como punto de partida. Esto es una clara muestra de la valoración que él mismo daba a su obra al considerarla el punto de inicio de la filosofía trascendental. 
al primero se tiene una representación de las cosas tal y como ellas aparecen; conforme al segundo una representación de tal y como ellas son. ${ }^{9}$

En esta doble tarea de establecer los principios formales del conocimiento sensible y del conocimiento inteligible Kant regresa nuevamente a una reflexión acerca del espacio y, junto con ello, a una nueva evaluación de la relación de los sólidos que son idénticos en todas sus partes pero que no son congruentes. Los principios formales del mundo sensible contienen la razón del vínculo por medio del cual las sustancias y sus estados se ligan entre sí en tanto que objetos de la sensibilidad; para Kant estos principios formales del mundo sensible, que como tales sólo pueden ser principios subjetivos, son dos: el espacio y el tiempo. El conocimiento sensible se basa en la percepción que de las cosas externas se tiene; dicha percepción se despliega en el espacio y el tiempo, pero ella no los crea sino que, por el contrario, requiere de su existencia previa. Esto no significa, empero, la existencia de un espacio y un tiempo para cada percepción de las cosas sensibles, ya que Kant considera que la existencia del espacio y del tiempo es singular y única: bajo la multiplicidad de regiones espaciales o de instantes de tiempo no hay sino secciones diferentes de un espacio y un tiempo únicos.

Con base en este doble carácter del espacio, su unicidad y el hecho de ser la forma fundamental de toda sensación, Kant concluye que el espacio es una intuición pura, anterior y condición de posibilidad de toda sensación y de toda experiencia. Kant asegura que esta intuición puede ser captada fácilmente a través de los axiomas de la geometría y de las construcciones (mentales) de los postulados. ${ }^{10}$ Ninguno de ellos es deducible de algún principio o noción universal acerca del espacio; más bien

${ }^{9}$ Dissertatio, sección II, $\S 4$.

${ }^{10}$ Kant señala, a manera de ejemplo, los siguientes axiomas:

1. El espacio no tiene más de tres dimensiones.

2. Entre dos puntos existe una única recta.

3. Por un punto en un plano es posible trazar un círculo con una recta dada. 
se trata de proposiciones que en él mismo se pueden discernir y ver. Así, para Kant, la evidencia de las demostraciones geométricas que se siguen de los axiomas, — evidencia concebida como la expresión clara de un conocimiento certero derivado de los sentidos- es la única posible, ya que por la manera en la que la geometría contempla las relaciones espaciales, en cuyo concepto está contenida la forma de toda intuición sensible, no hay nada que pueda ser percibido claramente si no es a través de la intuición, tal y como ella la contempla. En sus demostraciones, la geometría no procede concibiendo sus objetos a través de conceptos universales, sino que los coloca ante los ojos mediante una intuición singular, como sucede con los objetos sensibles.

Pero junto con los axiomas, algunas otras relaciones entre las figuras en el espacio tienen esta doble propiedad de no ser lógicamente deducibles y de ser igualmente evidentes; la relación entre los sólidos similares pero incongruentes [solidis perfecte similibus atque aequalibus, sed discongruentibus] es para Kant el ejemplo más claro:

No es sino a través de una intuición que se puede caracterizar la diversidad, a saber la de la imposibilidad de la congruencia. De ahí que la geometría use principios que no son solamente indudables y discursivos, sino que caen bajo la mirada de la mente. ${ }^{11}$

Nada de lo que se pueda enunciar discursivamente permitiría establecer la distinción entre ellas, ya que ésta es precisamente su característica primordial: son indistinguibles en cada una de sus partes; la diferencia interna que hace imposible la congruencia entre ellas sólo puede ser captada a través de la intuición.

Es importante señalar que la última proposición corresponde al postulado III de los Elementos de Euclides, mientras que la segunda corresponde a una versión modificada del postulado I y la primera no corresponde a ningún Postulado o Noción Común. Es claro que Kant los considera a todos como axiomas de la geometría (euclidiana) pero nos parece claro también, a propósito de este breve listado de axiomas, que la referencia de Kant a la geometría no es extraída directamente del texto euclidiano.

${ }^{11}$... sed sub obtutum mentis cadentibus. Kant, Dissertatio, sección III, $\S 15$. 
De este modo puede decirse que si bien en el texto de 1768 Kant aseguraba que no son las situaciones de las partes de los cuerpos las que determinan las condiciones del espacio, sino que más bien dependen de éstas, dos años después, en la Dissertatio, la condición del espacio como una intuición pura es concebida como la condición que hace posible la comprensión de la diferencia que existe entre dos cuerpos similares y no congruentes.

Como lo señalamos en un principio, en los Prolegómenos de 1783, Kant regresa por tercera ocasión al análisis de las parejas incongruentes. El problema general planteado en este texto es, como se sabe, el mismo que fue presentado en la Crítica de la razón pura: a condición de qué puede la metafísica devenir una ciencia. Parte de la estrategia de Kant para encontrar el camino hacia una respuesta a este problema se deriva de la que a su vez se puede dar a la pregunta acerca de cómo es que la matemática es una ciencia pura. En la terminología propia de la filosofía crítica, esta pregunta se formula de la siguiente manera: ¿cómo son posibles los juicios sintéticos a priori en matemáticas?

Para Kant, el conocimiento matemático no descansa sobre ningún principio de la experiencia, sino que es producto puro de la razón cuya certeza es completamente apodíctica. Se trata de un conocimiento que presenta sus conceptos en la intuición; pero si el conocimiento matemático es también a priori ello se debe a que sus conceptos se construyen en la intuición pura y en ello difiere de los conocimientos sintéticos que se alcanzan mediante la construcción de los conceptos a partir de los objetos de la intuición empírica, conocimientos que en este caso sólo son a posteriori. Así, el conocimiento matemático requiere, como condición de posibilidad para su existencia, una intuición pura en la que sea posible la construcción de sus conceptos. La respuesta a la pregunta inicial es entonces, según Kant, la respuesta acerca de cuál es esta intuición pura sobre la que la matemática construye sus conceptos. Pero si la intuición es una representación que depende de la presencia del objeto, Kant se pregunta cómo es posible la intuición de algo a priori. Una intuición pura tiene lugar en ausencia de un objeto con el cual ella 
se relaciona; se trata de la intuición de un objeto que precede al objeto, lo que únicamente es posible si contiene la forma de la sensibilidad que en la subjetividad precede a toda impresión real con la que el objeto la afecta. ${ }^{12}$

Como ya lo había asegurado en la Dissertatio, Kant afirma que el espacio y el tiempo son las intuiciones puras sobre las que la matemática funda todos sus conocimientos y sus juicios; ésta es, en particular, la condición de posibilidad de que las construcciones geométricas puedan ser consideradas apodícticas y necesarias, aun si se efectúan sobre un objeto particular presente en la sensibilidad. Esto es lo que se revela a partir de la relación fundamental en geometría: la relación de igualdad. La proposición que establece que la igualdad de dos figuras se deriva de su coincidencia, cuando una de ellas se aplica sobre la otra, puede considerarse una proposición sintética que reposa sobre la intuición inmediata, pero ésta debe ser pura y a priori, pues de otro modo la proposición no podría tener el carácter de ser apodícticamente cierta y se trataría únicamente de una proposición empírica. ${ }^{13}$

Todas las demostraciones de la igualdad plena [durchgängiger Gleichheit] de dos figuras dadas (de modo que todas las partes de una pueden ser colocadas en el lugar ocupado por las de la otra) se reducen a su coincidencia mutua [sie einander decken]; lo cual no es sino una proposición sintética que se apoya sobre la intuición inmediata [unmittelbaren Anschauung]; y esta intuición debe darse de manera pura y a priori, pues de otro modo dicha proposición no podría ser tomada como una proposición apodícticamente cierta y sólo tendría una certeza empírica. ${ }^{14}$

Sin embargo, a partir de esta primera proposición que establece la igualdad entre las figuras, se plantea necesariamente la pregunta de si la proposición que establece la relación entre dos

${ }^{12}$ Prolegómenos, §§8-9.

${ }^{13} \mathrm{El}$ criterio de igualdad por coincidencia bajo superposición se establece en la geometría de Euclides a través de una Noción Común (véase más adelante), pero en los Elementos no es una proposición que pueda demostrarse.

${ }^{14}$ Prolegómenos, § 12. 
figuras perfectamente idénticas, sin ninguna diferencia interna, que no se pueden encerrar en los mismos límites y que por lo tanto no coinciden cuando una de ellas se aplica sobre la otra, tiene igualmente el carácter de ser una proposición sintética. La imposibilidad de que el entendimiento pueda dar cuenta de la diferencia interna que impide la congruencia entre los dos objetos, a pesar de la coincidencia en cada una de sus partes internas, es para Kant la prueba clara de que esta propiedad sólo puede ser explicada en el ámbito de la intuición mediante sus relaciones externas en el espacio. Los objetos incongruentes se presentan como intuiciones sensibles, es decir, como apariencias, y en esta condición adquieren ese carácter: no son representaciones de objetos tal y como éstos son en sí mismos y tal y como el entendimiento los podría conocer. La determinación interna de una región del espacio - la región ocupada por la figura - sólo es posible mediante la determinación de su relación externa con todo el espacio - del que ella es una parte-, pero a su vez esta doble determinación sólo es posible a través de la sensibilidad, ya que el espacio es la forma de la intuición externa de la sensibilidad. Con el entendimiento, en cambio, no es posible dar cuenta de la relación de dos figuras simétricas y no congruentes: "Ningún concepto es capaz por sí mismo de permitirnos concebir la diferencia entre dos cosas que a pesar de ser semejantes e iguales resultan no congruentes, esto sólo podrá hacerse a través de las relaciones que proceden de la intuición." 15

De este modo el regreso de Kant al problema de las figuras similares e incongruentes en los Prolegómenos no busca dar cuenta de la relación en sí, sino del carácter sintético y a priori de la proposición que permite establecer la relación que guardan entre sí las dos figuras; el carácter y el fundamento de esta proposición es entonces el mismo que el de la proposición que establece la igualdad de las figuras.

Después de esta breve descripción de los tres momentos en los que Kant dirigió su atención al problema de la simetría de las figuras sólidas, vale la pena analizar con cierto detalle

${ }^{15}$ Prolegómenos, §13. 
el modo en el que este problema ha sido planteado desde el ámbito específico de la geometría. Para ello, además del texto de los Elementos de Euclides, nos referiremos principalmente a dos textos que por la forma de aproximarse al problema son de particular interés, amén de ser contemporáneos de la obra de Kant, se trata de la edición comentada del texto euclidiano preparada por Robert Simson (1756) y de los Éléments de Géométrie de Legendre, cuya primera edición data de 1794.

\section{La simetría en la geometría del espacio. El problema del ángulo sólido}

Sin duda en los Eléments de Géométrie de Legendre se encuentra el primer estudio explícito y detallado acerca del problema de la simetría de las figuras sólidas, aunque parece claro también que en algunos textos geométricos previos, como en la edición de Simson, este problema comienza ya a dibujarse y a ser tratado de manera indirecta. No pretendo extraer ninguna conclusión fácil sobre esta coincidencia temporal entre las reflexiones filosóficas de Kant y las reflexiones geométricas de Legendre, aun cuando creo que ésta debe mencionarse, y dirigiré la atención en cambio a rastrear los orígenes geométricos de este problema.

En el libro XI de los Elementos, Euclides introduce por primera vez un objeto geométrico, el ángulo sólido, el cual va a desempeñar un papel central en el problema de la simetría. Este nuevo objeto geométrico es definido de la siguiente manera:

$\boldsymbol{D}_{\mathrm{XI}-11}$ : Un ángulo sólido es la inclinación de más de dos líneas que se tocan entre sí y no están en la misma superficie con respecto a todas las líneas. O de otra forma: un ángulo sólido es el comprendido por más de dos ángulos planos construidos en el mismo punto, sin estar en el mismo plano.

Aparentemente esta definición intenta generalizar la definición del ángulo plano dada en el libro I, según la cual

$\boldsymbol{D}_{\text {I-8 }}$ : Un ángulo plano es la inclinación mutua de dos líneas en un plano que se encuentran y que no forman una línea recta. 
De acuerdo con ello, si un ángulo plano a $[B A C]$ está formado a partir de dos líneas que se cortan $-\mathrm{y}$ que por ello son coplanares- $\mathrm{L}[A B]$ y $\mathrm{L}[A C]$, un ángulo sólido $\mathfrak{A}[A]_{(B C D \ldots)}$ está formado a partir de las líneas $\mathrm{L}[A B], \mathrm{L}[A C], \mathrm{L}[A D], \ldots$, de las cuales no hay tres que sean coplanares, o bien por los ángulos planos $\mathfrak{a}[B A C], \mathfrak{a}[C A D], \ldots{ }^{16}$ De igual modo se puede decir, lo que resulta equivalente a la definición euclidiana, que el ángulo sólido $\mathfrak{A}[A]_{(B C D \ldots)}$ está formado a partir de la intersección de los planos $\Pi_{B A C}, \Pi_{C A D}, \ldots{ }^{17}$

A pesar de esta aparente analogía entre el ángulo plano y el ángulo sólido, el autor de los Elementos se enfrenta rápidamente a la imposibilidad de tratar a un ángulo sólido como una cantidad geométrica, tal y como lo hace con el ángulo plano. El tratamiento del ángulo plano como una cantidad se da a partir de la proposición 9 y hasta la proposición 23 del libro I. De este grupo de proposiciones destacan, entre otras, la construcción y caracterización del ángulo recto, la proposición I-16 en la que por primera vez se establece de manera clara una comparación cuantitativa entre ángulos, y desde luego la proposición I-23, en la cual Euclides se propone la construcción de un ángulo rectilíneo que sea igual a un ángulo rectilíneo dado. En todas ellas y en los Postulados (A $\iota \tau \eta \mu \alpha \tau \alpha)$, las Nociones Comunes $(\mathrm{K} o \iota \nu \alpha \iota \mathrm{E} \nu \nu \circ \iota \alpha \iota)$ desempeñan un papel fundamental, particularmente aquellas que proporcionan los criterios de igualdad y desigualdad entre cantidades: la Noción Común 7 (NC.7) que asegura que las cosas que coinciden entre sí son iguales entre sí y la Noción Común 8 (NC.8) que establece que el todo es mayor que la parte. La proposición XI-26, que se plantea la construcción de un ángulo sólido igual a un ángulo sólido dado, en una línea dada — que es la proposición análoga a la proposición I-23-, merece atención especial para comprender

\footnotetext{
${ }^{16}$ En adelante denotaré con $\mathfrak{a}[B A C]$ al ángulo plano cuyo vértice está en el punto $A$ y que está formado por las líneas $\mathrm{L}[A B]$ y $\mathrm{L}[A C]$; con $\mathfrak{A}[A]_{(B C D \ldots)}$ a un ángulo sólido formado por las líneas $\mathrm{L}[A B], \mathrm{L}[A C], \mathrm{L}[A D], \ldots \mathrm{y}$ cuyo vértice se encuentra en el punto $A$.

${ }^{17} \Pi_{B A C}$ denota al plano en donde yacen los puntos $B, A$ y $C$; de manera equivalente es el plano definido por las dos líneas $\mathrm{L}[A B]$ y $\mathrm{L}[A C]$ que se encuentran en el punto $A$.
} 
la dificultad a la que nos referimos. Como en todo problema, esta proposición exige tanto la construcción $(\kappa \alpha \tau \alpha \sigma \kappa \varepsilon v \eta)$ de la figura o la magnitud geométrica que se pide, como la prueba $(\alpha \tilde{\alpha} \delta \delta \varepsilon \iota \iota \varsigma)$ de que la construcción realizada es efectivamente la construcción requerida. Como lo veremos, Euclides resuelve parcialmente el problema de la construcción de un ángulo sólido, pero en lo que se refiere a la prueba de que el ángulo sólido que se ha construido es igual al ángulo sólido dado, se enfrenta al hecho de que si $N C .7$ y $N C .8$ proporcionan un criterio absoluto para la comparación — de igualdad y de desigualdad - entre dos ángulos planos, no pueden ser igualmente utilizadas en el caso de los ángulos sólidos. ${ }^{18}$

Si bien en la proposición XI-26 se pide la construcción de un ángulo sólido que sea igual a un ángulo sólido cualquiera, el autor de los Elementos considera sólo el caso en el que un ángulo sólido dado está constituido por tres ángulos planos: si $\mathfrak{A}[A]_{(B C D)}$ es el ángulo sólido dado con vértice en el punto $A$, formado por las líneas $\mathrm{L}[A B], \mathrm{L}[A C]$ y $\mathrm{L}[A D]-\mathrm{o}$ bien por los ángulos planos $\mathfrak{a}[B A C], \mathfrak{a}[C A D]$ y $\mathfrak{a}[B A D]-$, la construcción propuesta sugiere: primero trazar, a partir del punto $D$ que no es coplanar con los puntos $A, B$ y $C$, una línea $\mathrm{L}[D E]$ que sea

${ }^{18}$ Para comprender cabalmente este problema podemos definir - lo que claramente no es muy fiel al espíritu euclidiano, aun cuando sea geométricamente correcto- la magnitud de un ángulo plano a $[B A C]$ como la longitud del arco de la circunferencia unitaria, centrada en el punto $A$, acotada por los dos puntos en los que esta circunferencia se intersecará con las líneas $\mathrm{L}[A B]$ y L $[A C]$. Está claro que si dos ángulos planos son iguales de acuerdo con esta definición, entonces, en caso de poder transportar uno sobre el otro, ellos coincidirán plenamente, y se reencontrará así el criterio de igualdad derivado de NC.7. Del mismo modo, si uno de ellos es menor que el otro según esta misma definición, al ser transportado sobre el otro se presentará como parte de él, y reencontrará el criterio de desigualdad de $N C .8$. De manera análoga podemos definir la magnitud de un ángulo sólido $\mathfrak{a}[A]_{(B C D \ldots)}$ como el área de la sección de la esfera unitaria, centrada en el punto $A$, acotada por las líneas curvas definidas a partir de la intersección de la esfera con los planos $\Pi_{B A C}, \Pi_{C A D}, \ldots$ Podemos ver que el criterio de igualdad y desigualdad entre ángulos sólidos que se sigue de esta definición no coincide plenamente con la idea que se sigue de $N C .7$ y $N C .8$. En la última sección regresaré a esta representación de un ángulo sólido y veremos la ventaja que brinda para la comprensión de la relación de simetría. 
perpendicular al plano en el que se encuentran los puntos $A, B$ y $C .{ }^{19}$ Acto seguido se trazan las líneas $\mathrm{L}[D B]$ y $\mathrm{L}[D C]$.

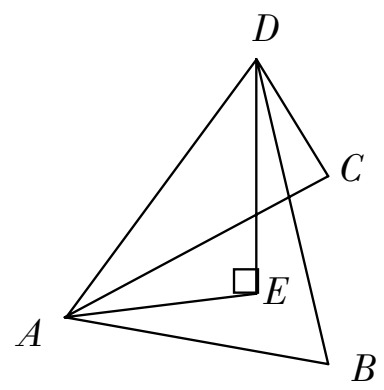

Figura 1

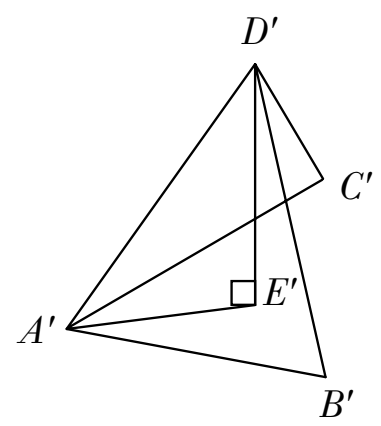

Figura $1^{\prime}$

Para la construcción de un ángulo sólido que sea igual a $\mathfrak{A}[A]_{(B C D)}$, a partir de una línea dada $\mathrm{L}\left[A^{\prime} B^{\prime}\right]$, Euclides propone primero tomar esta línea igual en longitud a la línea $\mathrm{L}[A B]$, y a partir de ella construir los ángulos planos $\mathfrak{a}\left[B^{\prime} A^{\prime} C^{\prime}\right]$ y $\mathfrak{a}\left[B^{\prime} A^{\prime} E^{\prime}\right]$ de modo que sean iguales, respectivamente, a los ángulos planos $\mathfrak{a}[B A C]$ y $\mathfrak{a}[B A E] .{ }^{20}$ Estos dos ángulos se construyen del mismo lado de la línea $\mathrm{L}\left[A^{\prime} B^{\prime}\right]$. La línea $\mathrm{L}\left[A^{\prime} E^{\prime}\right]$ se toma de la misma longitud que la línea $\mathrm{L}[A E]$ y en el punto $E^{\prime}$ del plano que contiene a los puntos $B^{\prime}, A^{\prime}$, y $C^{\prime}$ se levanta una línea $\mathrm{L}\left[E^{\prime} D^{\prime}\right]$ perpendicular a este plano y de la misma longitud que $\mathrm{L}[E D] .{ }^{21}$ De esta manera se ha construido el ángulo sólido $\mathfrak{A}\left[A^{\prime}\right]_{\left(B^{\prime} C^{\prime} D^{\prime}\right)}$, formado a partir del punto $A^{\prime}$ por las líneas $\mathrm{L}\left[A^{\prime} B^{\prime}\right], \mathrm{L}\left[A^{\prime} C^{\prime}\right]$ y $\mathrm{L}\left[A^{\prime} D^{\prime}\right]$. Una vez que el ángulo sólido $\mathfrak{A}\left[A^{\prime}\right]_{\left(B^{\prime} C^{\prime} D^{\prime}\right)}$ ha sido construido, Euclides hace ver que los triángulos $\mathrm{T}[A B D]$ y

${ }^{19}$ Construcción dada en la proposición 11 del libro XI;

${ }^{20}$ Estas construcciones están justificadas por Euclides en las proposiciones 2 y 23 del libro I. Es importante notar que estas proposiciones se pueden aplicar a este caso en virtud de que los ángulos a $[B A C]$ y $\mathfrak{a}[B A E]$ son coplanares y los ángulos construidos a $\left[B^{\prime} A^{\prime} C^{\prime}\right]$ y a $\left[B^{\prime} A^{\prime} E^{\prime}\right]$ también lo serán.

${ }^{21}$ Según la proposición 12 del libro XI. 
$\mathrm{T}\left[A^{\prime} B^{\prime} D^{\prime}\right]$ son congruentes, al igual que los triángulos $\mathrm{T}[A C D]$ y $\mathrm{T}\left[A^{\prime} C^{\prime} D^{\prime}\right]$ así como $\mathrm{T}[A B C]$ y $\mathrm{T}\left[A^{\prime} B^{\prime} C^{\prime}\right] .{ }^{22}$ De estas congruencias de triángulos puede asegurar la igualdad de los ángulos correspondientes: $\mathfrak{a}[B A C]=\mathfrak{a}\left[B^{\prime} A^{\prime} C^{\prime}\right], \mathfrak{a}[B A D]=\mathfrak{a}\left[B^{\prime} A^{\prime} D^{\prime}\right]$ y $\mathfrak{a}[C A D]=\mathfrak{a}\left[C^{\prime} A^{\prime} D^{\prime}\right]$.

En realidad si bien es cierto que Euclides ha presentado y justificado todos los pasos de la construcción $(\kappa \alpha \tau \alpha \sigma \kappa \varepsilon v \eta)$, no sucede lo mismo con la prueba $(\alpha \dot{\alpha} \delta \delta \varepsilon \iota \xi \iota \varsigma)$, ya que simplemente se limita a señalar la igualdad de los ángulos planos, como si a partir de ésta se pudiese concluir la igualdad de los ángulos sólidos. Para poder concluir la igualdad $\mathfrak{A}[A]_{(B C D)}=$ $\mathfrak{A}\left[A^{\prime}\right]_{\left(B^{\prime} C^{\prime} D^{\prime}\right)}$ a partir de las igualdades $\mathfrak{a}[B A C]=\mathfrak{a}\left[B^{\prime} A^{\prime} C^{\prime}\right]$, $\mathfrak{a}[B A D]=\mathfrak{a}\left[B^{\prime} A^{\prime} D^{\prime}\right]$ y $\mathfrak{a}[C A D]=\mathfrak{a}\left[C^{\prime} A^{\prime} D^{\prime}\right]$, sería necesaria una proposición más general que se podría enunciar de la siguiente manera:

$\boldsymbol{A}_{1}$ : Dos ángulos sólidos formados cada uno por tres ángulos planos y que son respectivamente iguales dos a dos, son iguales entre sí.

El autor de los Elementos no proporciona ninguna demostración previa de la proposición $\boldsymbol{A}_{1}$, es decir, que si suponemos que la igualdad $\mathfrak{A}[A]_{(B C D)}=\mathfrak{A}\left[A^{\prime}\right]_{\left(B^{\prime} C^{\prime} D^{\prime}\right)}$ se apoya en ella, habremos de concluir que la proposición XI-26 se sigue de una proposición que no ha sido demostrada.

Pero la igualdad $\mathfrak{A}[A]_{(B C D)}=\mathfrak{A}\left[A^{\prime}\right]_{\left(B^{\prime} C^{\prime} D^{\prime}\right)}$ se podría deducir, en caso de que los cuerpos sólidos $\mathrm{C}[A B C D]$ y $\mathrm{C}\left[A^{\prime} B^{\prime} C^{\prime} D^{\prime}\right]$ fuesen iguales, a partir del hecho de que se trata de los ángulos sólidos correspondientes en dichos cuerpos. ${ }^{23}$

En este caso es la definición $\boldsymbol{D}_{\mathrm{XI}-10}$ introducida por Euclides la que permite concluir que estas figuras sólidas son iguales y, por lo tanto, lo son los ángulos sólidos: ${ }^{24}$

\section{${ }^{22}$ Proposiciones 4 y 8 del libro I.}

${ }^{23}$ La prueba entonces sería similar a la que Euclides utiliza para demostrar la igualdad de dos ángulos planos, en la que hace ver que se trata de ángulos correspondientes en dos triángulos que, de acuerdo con $N C .7$, son iguales.

${ }^{24} \mathrm{La}$ definición de una igualdad no es inédita en el texto de Euclides, pero 
$\boldsymbol{D}_{\mathrm{XI}-10}$ : Las figuras sólidas iguales y semejantes son aquellas que están comprendidas por planos semejantes e iguales en número y magnitud.

Según esta definición, la igualdad C $[A B C D]=\mathrm{C}\left[A^{\prime} B^{\prime} C^{\prime} D^{\prime}\right]$ se sigue de las igualdades de los triángulos $\mathrm{T}[A B D]=\mathrm{T}\left[A^{\prime} B^{\prime} D^{\prime}\right]$, $\mathrm{T}[A C D]=\mathrm{T}\left[A^{\prime} C^{\prime} D^{\prime}\right]$ y $\mathrm{T}[A B C]=\mathrm{T}\left[A^{\prime} B^{\prime} C^{\prime}\right]$, a las que se puede añadir la igualdad $\mathrm{T}[B C D]=\mathrm{T}\left[B^{\prime} C^{\prime} D^{\prime}\right]$. Este segundo argumento parece estar más sólidamente fundamentado en la estructura lógica de los Elementos; sin embargo, no deja de llamar la atención el silencio de Euclides en lo que se refiere al sustento principal de la prueba. La construcción euclidiana de $\mathfrak{A}\left[A^{\prime}\right]_{\left(B^{\prime} C^{\prime} D^{\prime}\right)}$ es simplemente la construcción de un ángulo sólido cuyos ángulos planos son iguales a los de $\mathfrak{A}[A]_{(B C D)}$, lo que permite pensar que su demostración se basa en la aceptación implícita de la proposición $\boldsymbol{A}_{1}$.

Un hecho notable es que tanto la proposición $\boldsymbol{A}_{1}$ como la definición $\boldsymbol{D}_{\mathrm{XI}-10}$ podrían servir igualmente como fundamento para la prueba de la igualdad del ángulo sólido $\mathfrak{A}[A]_{(B C D)}$ con el ángulo $\mathfrak{A}\left[A^{\prime}\right]_{\left(B^{\prime} C^{\prime \prime} D^{\prime \prime}\right)}$ que se obtiene si en la construcción anterior los ángulos planos $\mathfrak{a}\left[B^{\prime} A^{\prime} E^{\prime \prime}\right]$ y $\mathfrak{a}\left[B^{\prime} A^{\prime} C^{\prime \prime}\right]$, también iguales respectivamente a los ángulos planos $\mathfrak{a}[B A C]$ y a $\mathfrak{a} A B E]$, se trazan del otro lado de la línea $\mathrm{L}\left[A^{\prime} B^{\prime}\right]$. Esto significa que esta línea $\mathrm{L}\left[A^{\prime} B^{\prime}\right]$ puede dividir el plano en dos regiones distintas y que en una de ellas tiene lugar la construcción de los ángulos $\mathfrak{a}\left[B^{\prime} A^{\prime} E^{\prime \prime}\right]$ y $\mathfrak{a}\left[B^{\prime} A^{\prime} C^{\prime \prime}\right]$, y en la otra la de los ángulos $\mathfrak{a}\left[B^{\prime} A^{\prime} E^{\prime}\right]$ y $\mathfrak{a}\left[B^{\prime} A^{\prime} C^{\prime}\right]{ }^{25} \mathrm{Al}$ seguir paso a paso la misma construcción,

no por ello deja de ser problemática. El ejemplo más célebre es el de la definición 5 del libro $\mathrm{V}$, donde Euclides introduce lo que habrá de entenderse por la igualdad de dos razones, definición que por ello supone la presencia de cuatro magnitudes que serán necesariamente homogéneas por parejas.

${ }^{25}$ Euclides asume la hipótesis de que una línea divide un plano en dos regiones distintas en varias proposiciones de los Elementos; dos ejemplos notables son el de la proposición I-7 y el de la proposición I-22, la cual presenta la misma situación que la que aquí describimos para la geometría del espacio. Hilbert considera ( $c f r$. Grundlagen der Geometrie) que esta hipótesis es en realidad una proposición que puede ser demostrada a partir de los axiomas de incidencia y de orden. 
el ángulo sólido $\mathfrak{A}\left[A^{\prime}\right]_{\left(B^{\prime} C^{\prime \prime} D^{\prime \prime}\right)}$ y el cuerpo sólido $\mathrm{C}\left[A^{\prime} B^{\prime} C^{\prime \prime} D^{\prime \prime}\right]$ así obtenidos no son los mismos que los que se obtienen en la figura $1^{\prime}$ :

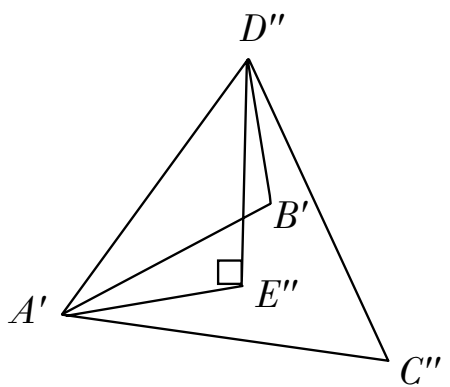

Figura $1^{\prime \prime}$

Los tres ángulos planos que constituyen el ángulo sólido $\mathfrak{A}\left[A^{\prime}\right]_{\left(B^{\prime} C^{\prime \prime} D^{\prime \prime}\right)}$ son iguales a los ángulos planos que forman el ángulo sólido $\mathfrak{A}\left[A^{\prime}\right]_{\left(B^{\prime} C^{\prime} D^{\prime}\right)}$, pero el orden en la orientación de estos ángulos a partir del vértice $A^{\prime}$ es distinto, lo que provoca al mismo tiempo que la disposición de las caras de los cuerpos sólidos C $[A B C D]$ y $\mathrm{C}\left[A^{\prime} B^{\prime} C^{\prime \prime} D^{\prime \prime}\right]$ sea diferente. Al comparar la disposición de los ángulos planos en los ángulos sólidos $\mathfrak{A}[A]_{(B C D)}$ y $\mathfrak{A}\left[A^{\prime}\right]_{\left(B^{\prime} C^{\prime \prime} D^{\prime \prime}\right)}$, podemos observar que en el primero se tiene, en sentido levógiro, el orden $\mathfrak{D}[\mathfrak{a}[B A C]$, $\mathfrak{a}[B A D]$, $\mathfrak{a}[C A D]]$, mientras que en el segundo el orden se presenta de manera invertida $\mathfrak{D}\left[\mathfrak{a}\left[B^{\prime} A^{\prime} C^{\prime \prime}\right], \mathfrak{a}\left[C^{\prime \prime} A^{\prime} D^{\prime \prime}\right], \mathfrak{a}\left[B^{\prime} A^{\prime} D^{\prime \prime}\right]\right]$.

Otra proposición de los Elementos que nos lleva a una conclusión similar es la proposición 28 del mismo libro XI y que, aparentemente, no hace sino generalizar para la geometría del espacio lo que la proposición I-34 establece para la geometría del plano. ${ }^{26}$ La proposición XI-28 asegura que un plano que

${ }^{26}$ La proposición I-34 asegura que el área de un paralelogramo será dividida en dos partes iguales por la diagonal del paralelogramo. Es importante 
corta a un paralelepípedo a través de las diagonales de dos lados opuestos divide a este paralelepípedo en dos prismas iguales. Esto significa que si C $[A B C D E F G H]$ es un paralelepípedo cuyas caras son los cuadriláteros opuestos $\mathrm{Q}[A B C D]$ y $\mathrm{Q}[E F G H]$, $\mathrm{Q}[A B E F]$ y $\mathrm{Q}[C D G H], \mathrm{Q}[A D E H]$ y $\mathrm{Q}[B C F G]$, entonces el plano $\Pi_{A D F G}$ divide al sólido $\mathrm{C}[A B C D E F G H]$ en dos prismas iguales $\mathrm{R}[A B C D F G]$ y $\mathrm{R}[A D E H F G]$ :

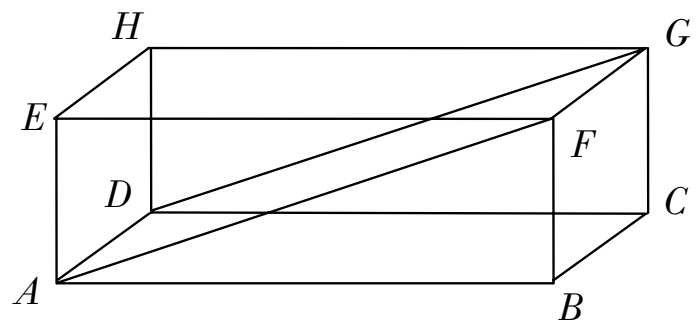

Figura 2

La prueba de Euclides asegura que las caras correspondientes de los prismas son iguales: $\mathrm{Q}[A B C D]=\mathrm{Q}[E F G H], \mathrm{T}[A B F]=$ $\mathrm{T}[A E F], \mathrm{Q}[A D E H]=\mathrm{Q}[B C F G], \mathrm{T}[D G H]=\mathrm{T}[D C G]$; igualdades que se obtienen sin ninguna dificultad. La igualdad de $\mathrm{R}[A B C D F G]$ y $\mathrm{R}[A D E H F G]$ se deduce entonces, y en este caso el autor de los Elementos es explícito, de la definición $\boldsymbol{D}_{\mathrm{XI}-10}$.

Aquí también se observa que en los prismas $\mathrm{R}[A B C D F G]$ y $\mathrm{R}[A D E H F G]$ el orden de los ángulos planos correspondientes a los ángulos sólidos $\mathfrak{A}[B]_{(A C F)}$ y $\mathfrak{A}[H]_{(G E D)}$, a partir de los vértices $B$ y $H$, es distinto. Lo mismo sucede con las demás parejas de ángulos sólidos correspondientes: $\mathfrak{A}[C]_{(D B G)}$ y $\mathfrak{A}[E]_{(F H A)}$, $\mathfrak{A}[A]_{(B D F)}$ y $\mathfrak{A}[G]_{(H F D)}, \mathfrak{A}[D]_{(C A G)}$ y $\mathfrak{A}[F]_{(H F D)}$.

señalar que Euclides demuestra que el paralelogramo queda dividido por la diagonal en dos triángulos congruentes. 
El punto que nos interesa aclarar en torno de estas dos proposiciones euclidianas se refiere al fundamento último de la relación de igualdad de las figuras sólidas. Hemos señalado que, de acuerdo con la estructura lógica del texto euclidiano, ésta se deriva de la definición $\boldsymbol{D}_{\mathrm{XI}-10}$, la que adquiere así el carácter de ser una proposición no demostrada que resulta imprescindible en la prueba $(\alpha \tilde{\alpha} \delta \varepsilon \varepsilon \iota \xi \iota \varsigma)$ de las dos proposiciones que hemos analizado. Pero al asegurar la igualdad de dos figuras sólidas a partir de la igualdad entre sus caras, la definición $\boldsymbol{D}_{\mathrm{XI}-10}$ adquiere también el estatus de un axioma que permite eliminar de la geometría del espacio la necesidad de que una de las figuras sea aplicada $(\hat{\epsilon} \varphi \alpha \rho \mu \delta ́ \zeta \epsilon \theta \alpha \iota)$ sobre otra a fin de poder probar su igualdad.

La necesidad de aplicar o superponer dos figuras planas para probar la igualdad entre ellas constituye uno de los puntos más delicados de la geometría euclidiana, ya que es evidente que ningún postulado, concebido propiamente como una cláusula constructiva, permite tal operación. ${ }^{27}$ Entonces me parece que el autor de los Elementos opta por no recurrir una segunda vez a esta operación y no sugiere, en ninguna de las dos proposiciones analizadas, que una figura sólida se aplique sobre otra. El uso explícito de la definición $\boldsymbol{D}_{\mathrm{XI}-10}$ en la proposición XI-28, o bien el uso implícito de esta definición o de la proposición $\boldsymbol{A}_{1}$ en XI-26, muestran que ha optado por una estrategia distinta, en la cual se busca extender a las figuras sólidas la conclusión de la superposición efectuada sobre figuras planas. Así, por ejemplo, en XI-28 la igualdad de los triángulos T $[A B F]$ y T $[A E F]$ (figura 2), se deduce de los teoremas de congruencia para triángulos de la geometría del plano, teoremas que se demuestran precisamente a partir de la superposición de estos triángulos. Si Euclides no utiliza en ningún momento la superposición de figuras en la geometría del espacio, el camino que sigue y que acabamos de señalar le permite vislumbrar que la igualdad de

${ }^{27}$ De las dificultades y problemas relacionados con esta operación geométrica de superposición o aplicación de figuras, en el caso de la geometría del plano ya hemos hablado en otro lugar, véase "Two Ways of Reasoning and Two Ways of Arguing in Geometry. Some Remarks Concerning the Application of Figures in Euclidean Geometry". 
las figuras sólidas no deberá entenderse necesariamente en el sentido de NC.7.

Pero si por un momento la operación geométrica de la superposición de figuras sólidas se supone válida, se podría constatar, a propósito de las figuras $1,1^{\prime}$ y $1^{\prime \prime}$, que las siguientes igualdades entre las figuras sólidas deben ser entendidas de manera diferente:

1. $\mathfrak{A}[A]_{(B C D)}=\mathfrak{A}\left[A^{\prime}\right]_{\left(B^{\prime} C^{\prime} D^{\prime}\right)}$ y $\mathrm{C}[A B C D]=\mathrm{C}\left[A^{\prime} B^{\prime} C^{\prime} D^{\prime}\right]$.

2. $\mathfrak{A}[A]_{(B C D)}=\mathfrak{A}\left[A^{\prime}\right]_{\left(B^{\prime} C^{\prime \prime} D^{\prime \prime}\right)}$ y $\mathrm{C}[A B C D]=\mathrm{C}\left[A^{\prime} B^{\prime} C^{\prime \prime} D^{\prime \prime}\right]$.

La primera de ellas se refiere a ángulos sólidos y figuras sólidas que, al suponer válido el que uno de ellos se pueda aplicar sobre el otro - de manera similar al procedimiento empleado por Euclides en la demostración de los teoremas de congruencia en la geometría plana-, coinciden plenamente. En este caso será posible decir que se trata de figuras congruentes: $\mathfrak{A}[A]_{(B C D)} \equiv \mathfrak{A}\left[A^{\prime}\right]_{\left(B^{\prime} C^{\prime} D^{\prime}\right)}$ y $\mathrm{C}[A B C D] \equiv \mathrm{C}\left[A^{\prime} B^{\prime} C^{\prime} D^{\prime}\right]$. Pero, en el segundo caso, los ángulos sólidos y las figuras sólidas no pueden coincidir al ser aplicados uno sobre el otro, a pesar de que se satisfacen las condiciones de la definición $\boldsymbol{D}_{\mathrm{XI}-10}$. Lo mismo sucede, en la figura 2 , con los prismas $\mathrm{R}[A B C D F G]$ y $\mathrm{R}[A D E H F G]$, cuya igualdad se deduce de esta definición a pesar de no ser congruentes. De este modo, la diferencia entre estos dos tipos de relación de igualdad, que la operación geométrica de superposición de figuras sólidas permitiría distinguir, queda eliminada con la definición $\boldsymbol{D}_{\mathrm{XI}-10}$.

Robert Simson parece ser el primero en señalar, en su edición de los Elementos (1756), el carácter polémico de la definición $\boldsymbol{D}_{\mathrm{XI}-10}$, al grado de que no duda en eliminarla del grupo de definiciones del libro XI y sugiere que se trata más bien de un teorema que debe ser demostrado. ${ }^{28}$ Esta misma actitud se en-

${ }^{28}$ De hecho para mostrar que en su formulación euclidiana la proposición $\boldsymbol{D}_{\mathrm{XI}-10}$ no es universalmente válida, Simson analiza el ejemplo de dos figuras sólidas que no resultan ser iguales a pesar de satisfacer las condiciones de esta definición. El ejemplo es interesante por la doble razón de que involucra también la igualdad de los ángulos sólidos: al tomar dos pirámides congruen- 
cuentra también en todas las ediciones de los Éléments de Géométrie de Legendre. Tanto para Simson como para Legendre, el desplazamiento rígido de figuras es una operación geométrica legítima, por lo que toda prueba de congruencia de dos figuras se lleva a cabo mediante la aplicación de una figura sólida sobre la otra. Pero si bien es cierto, como lo hemos señalado, que con el reconocimiento de esta aplicación se podrían distinguir claramente los dos tipos de igualdad por congruencia y por simetría, sólo Legendre trata de manera directa, a partir de este hecho, el tema de la relación de simetría en el espacio.

El punto de partida de su estudio, y a partir del cual justifica el hecho de que la relación de simetría es una modalidad de la igualdad que no puede ser identificada con la congruencia, es la demostración de la proposición $\boldsymbol{A}_{1}$. Legendre asegura que para dos ángulos triedros —ángulos sólidos formados por tres ángulos planos - cuyos ángulos planos son iguales dos a dos, los ángulos formados por los planos en los que están esos án-

tes, en general pueden ser cualesquiera dos poliedros congruentes, en una de las caras de uno de ellos añade una pirámide más pequeña, y en la pirámide congruente resta una pirámide igual a la anterior:

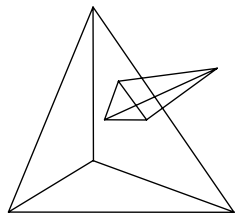

Figura 3

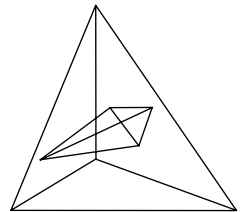

Figura $3^{\prime}$

El añadido de la figura 3 es igual (congruente) al hueco de la figura $3^{\prime}$; está claro que las figuras no podrán ser iguales y, sin embargo, las caras que las forman son iguales dos a dos. Más aún, si los ángulos sólidos considerados hasta ahora en una figura son aquellos que se toman desde el interior del cuerpo, los dos ángulos sólidos formados por la pirámide entrante y saliente están formados por ángulos planos que también son iguales dos a dos, y claramente distintos ya que son "complementos" el uno del otro. Así, el ejemplo de Simson sirve para poner en tela de juicio precisamente las dos proposiciones que han servido como apoyo para las pruebas de Euclides. 
gulos planos son también iguales. Si en las figuras 1 y $\mathbf{l}^{\prime}$ se consideran los ángulos sólidos $\mathfrak{A}[A]_{(B C D)}$ y $\mathfrak{A}\left[A^{\prime}\right]_{\left(B^{\prime} C^{\prime} D^{\prime}\right)}$, formados respectivamente por los ángulos planos $\mathfrak{a}[B A C], \mathfrak{a}[C A D]$ y $\mathfrak{a}[B A D]$ y por los ángulos planos $\mathfrak{a}\left[B^{\prime} A^{\prime} C^{\prime}\right], \mathfrak{a}\left[C^{\prime} A^{\prime} D^{\prime}\right]$ y $\mathfrak{a}\left[B^{\prime} A^{\prime} D^{\prime}\right]$, los cuales son iguales por parejas, y si el ángulo formado por los planos $\Pi_{B A D}$ y $\Pi_{C A D}$ se denota como $\mathfrak{a}_{A D}-\mathrm{el}$ ángulo en la arista $\mathrm{L}[A D]-$, entonces el teorema de Legendre asegura que si en los ángulos sólidos $\mathfrak{A}[A]_{(B C D)}$ y $\mathfrak{A}\left[A^{\prime}\right]_{\left(B^{\prime} C^{\prime} D^{\prime}\right)}$ se satisfacen las igualdades:

$\mathrm{I}: \mathfrak{a}[B A C]=\mathfrak{a}\left[B^{\prime} A^{\prime} C^{\prime}\right], \mathfrak{a}[C A D]=\mathfrak{a}\left[C^{\prime} A^{\prime} D^{\prime}\right]$ y $\mathfrak{a}[B A D]=$ $\mathfrak{a}\left[B^{\prime} A^{\prime} D^{\prime}\right]$,

entonces se siguen las igualdades

$$
\text { II: } \mathfrak{a}_{A D}=\mathfrak{a}_{A^{\prime} D^{\prime}}, \mathfrak{a}_{A B}=\mathfrak{a}_{A^{\prime} B^{\prime}}, \text { y } \mathfrak{a}_{A C}=\mathfrak{a}_{A^{\prime} C^{\prime}} .
$$

Legendre puede asegurar a partir de I y II, que si se aplica el ángulo $\mathfrak{A}[A]_{(B C D)}$ sobre el ángulo $\mathfrak{A}\left[A^{\prime}\right]_{\left(B^{\prime} C^{\prime} D^{\prime}\right)}$, ambos coinciden, por lo que son congruentes: $\mathfrak{A}[A]_{(B C D)} \equiv \mathfrak{A}\left[A^{\prime}\right]_{\left(B^{\prime} C^{\prime} D^{\prime}\right)}$. El razonamiento que le permite concluir que II se sigue de I también le permite concluir, al comparar ahora las figuras 1 y $1^{\prime \prime}$, que si las siguientes igualdades se satisfacen:

$$
\begin{aligned}
& \mathrm{I}^{\prime}: \mathfrak{a}[B A C]=\mathfrak{a}\left[B^{\prime} A^{\prime} C^{\prime \prime}\right], \mathfrak{a}[C A D]=\mathfrak{a}\left[C^{\prime \prime} A^{\prime} D^{\prime}\right] \mathrm{y} \\
& \mathfrak{a}[B A D]=\mathfrak{a}\left[B^{\prime} A^{\prime} D^{\prime}\right]
\end{aligned}
$$

entonces también lo hacen las igualdades

$$
\text { III: } \mathfrak{a}_{A D}=\mathfrak{a}_{A^{\prime} D^{\prime}}, \mathfrak{a}_{A B}=\mathfrak{a}_{A^{\prime} B^{\prime}}, \text { y } \mathfrak{a}_{A C}=\mathfrak{a}_{A^{\prime} C^{\prime \prime}}
$$

Pero Legendre concluye que si bien a partir de I' y III se puede asegurar la congruencia de cada uno de los ángulos planos que componen al ángulo sólido $\mathfrak{A}[A]_{(B C D)}$ con el ángulo plano correspondiente en el ángulo sólido $\mathfrak{A}\left[A^{\prime}\right]_{\left(B^{\prime} C^{\prime \prime} D^{\prime \prime}\right)}$, no es posible 
superponer o aplicar uno de estos ángulos sobre el otro debido a la diferencia en la orientación de los ángulos planos a partir de los vértices $A$ y $A^{\prime}$. La relación de simetría entre estos ángulos, $\mathfrak{A}[A]_{(B C D)} \cong \mathfrak{A}\left[A^{\prime}\right]_{\left(B^{\prime} C^{\prime \prime} D^{\prime \prime}\right)}$, queda así determinada no a partir de las magnitudes geométricas sino a partir del modo en el que ellas se ordenan en el espacio. Es de nuevo esta diferencia en la disposición de los ángulos planos (como se observa entre las figuras 1 y $1^{\prime \prime}$ ) la que impide la coincidencia de las dos figuras, por lo que Legendre caracteriza la relación $\mathfrak{A}[A]_{(B C D)} \cong$ $\mathfrak{A}\left[A^{\prime}\right]_{\left(B^{\prime} C^{\prime} D^{\prime \prime}\right)}$ como una igualdad que "no es absoluta y que por lo tanto merece ser denominada de manera particular: la igualdad por simetría". ${ }^{29}$

A partir de esta relación de simetría de dos ángulos sólidos triedros, Legendre entiende que un ángulo sólido es simétrico a un ángulo dado $\mathfrak{A}[A]_{(B C D \ldots)}$, formado por los ángulos planos $\mathfrak{a}[B A C], \mathfrak{a}[C A D], \ldots$, si es congruente con aquel que se obtiene al prolongar todas y cada una de las aristas $\mathrm{L}[A B]$, $\mathrm{L}[A C], \ldots$ hacia el otro lado del vértice $A$. De este modo se entiende que un ángulo simétrico a $\mathfrak{A}[A]_{(B C D \ldots)}$ es congruente con el ángulo sólido $\mathfrak{A}[A]_{\left(B^{\prime} C^{\prime} D^{\prime} \ldots\right)}$, formado por los ángulos planos $\mathfrak{a}\left[B^{\prime} A C^{\prime}\right], \mathfrak{a}\left[C^{\prime} A D^{\prime}\right], \ldots$, que son claramente iguales a los ángulos a $[B A C], \mathfrak{a}[C A D], \ldots, \mathrm{y}$ en donde el punto $A$ es siempre un punto interior a las líneas $\mathrm{L}\left[B B^{\prime}\right], \mathrm{L}\left[C C^{\prime}\right], \ldots$ Una vez establecida así la relación de simetría para los ángulos sólidos, Legendre introduce una definición general para la relación de simetría entre figuras sólidas:

$\boldsymbol{D}_{S}$ : Dos poliedros son simétricos si tienen una base común y son construidos de manera similar, uno por encima y el otro por debajo del plano de la base y con la condición de que los vértices de los ángulos sólidos homólogos se encuentren situados a distancias iguales del plano de la base y sobre una misma línea perpendicular a ese plano.

Para Legendre es evidente que todo poliedro que sea congruente con uno de los dos poliedros así relacionados será ne-

${ }^{29}$ A.M. Legendre, Éléments de Géométrie, libro V, proposición XIII. 
cesariamente simétrico al otro. ${ }^{30}$ Es importante señalar que no hay en esta definición ninguna alusión a la relación que entre sí deben guardar las magnitudes geométricas, ni a las figuras planas que forman sus caras, ni a los respectivos ángulos sólidos que componen a los dos poliedros simétricos.

Pero la prueba de que la relación de simetría así definida es una forma de la igualdad descansa sobre dos hechos: que cada una de las magnitudes planas que constituyen a un poliedro es igual a la magnitud plana correspondiente en el poliedro simétrico y que dos poliedros simétricos tienen el mismo contenido espacial o volumen. La primera propiedad se puede enunciar de acuerdo con la siguiente proposición:

$\boldsymbol{P}_{2}$ : En dos poliedros simétricos las caras homólogas son iguales una a una y el ángulo formado por dos caras adyacentes en uno de ellos es igual al ángulo formado por las caras homólogas en el otro.

De acuerdo con esta proposición que asegura la congruencia de las caras homólogas de los poliedros simétricos, Legendre encuentra que el núcleo de la relación de simetría entre ellos, y el fundamento de la imposibilidad de su congruencia, yace en la relación que existe entre los respectivos ángulos sólidos:

$\boldsymbol{P}_{3}$ : Los ángulos sólidos correspondientes de dos poliedros simétricos son ángulos sólidos simétricos.

Al ser simétricos entre sí en los ángulos sólidos homólogos se establecen los límites de la relación de congruencia: cada magnitud plana es congruente con la respectiva magnitud plana del poliedro simétrico, lo mismo que los ángulos que forman entre sí, pero al conjuntarse en cada vértice y formar un ángulo sólido, la orientación que en él toman hace imposible la congruencia de los ángulos sólidos y, por lo tanto, de los poliedros.

\footnotetext{
${ }^{30}$ Legendre puede concluir así la unicidad de la figura simétrica, ya que si se toma una tercera figura que sea simétrica con la primera, pero tomando como base común otra cara del primer poliedro, entonces este tercer poliedro será claramente congruente con el segundo.
} 
Entre dos figuras sólidas simétricas existe así una congruencia local, pero no es posible obtener una congruencia global. Las magnitudes congruentes son siempre magnitudes planas o lineales: aristas o ángulos planos, caras congruentes y ángulos entre ellas; pero el orden espacial en el cual se presentan para formar un cuerpo tridimensional es distinto en cada una de ellas, lo que impide la congruencia de los dos poliedros.

Pero si con la proposición $\boldsymbol{P}_{2}$ ha quedado demostrada una equivalencia cuantitativa local, queda por demostrar aún la igualdad cuantitativa global de dos poliedros sólidos, Legendre enuncia así la siguiente proposición:

$\boldsymbol{P}_{5}$ : Dos poliedros simétricos son equivalentes entre sí, es decir, tienen igual volumen.

Si en el caso de dos figuras planas no congruentes la geometría euclidiana estableció con éxito su equivalencia en área mediante la partición de éstas en el mismo número de partes congruentes - procedimiento que descansa necesariamente en el postulado $\mathrm{V}$-, en el caso de las figuras sólidas congruentes este camino se encuentra siempre limitado por la presencia de ángulos sólidos simétricos. Consciente de este problema, Legendre tiene que neutralizar el papel limitante del ángulo sólido para probar que todo poliedro es equivalente a su poliedro simétrico. La prueba de esta proposición $\boldsymbol{P}_{5}$ se deduce básicamente de la proposición que asegura que dos pirámides triangulares con bases equivalentes e igual altura son equivalentes:

$\boldsymbol{P}_{4}$ : Dos pirámides triangulares con bases equivalentes y alturas iguales son equivalentes.

La implicación $\boldsymbol{P}_{4} \rightarrow \boldsymbol{P}_{5}$ se sigue del hecho de que dos poliedros simétricos podrán descomponerse en el mismo número de pirámides triangulares que por parejas tienen bases iguales (congruentes) y alturas iguales. Las pirámides triangulares de los dos poliedros simétricos serán claramente simétricas entre sí, de modo que la estrategia general de Legendre es hacer ver que la equivalencia entre dos poliedros simétricos se deduce de 
la equivalencia entre las pirámides triangulares, también simétricas entre sí, en las que cada uno de ellos se puede dividir.

\section{La simetría y la intuición del espacio. El problema} de la superposición de las figuras

Como he señalado, al introducir la definición $\boldsymbol{D}_{\mathrm{XI}-10}$ el autor de los Elementos parece cerrar el paso a la posibilidad de establecer que la congruencia y la simetría son dos modalidades distintas de la igualdad en la geometría del espacio, toda vez que ella impide que la relación de simetría pueda pensarse de manera explícita en el marco de los libros XI y XII. Si, en la geometría del plano, dos de los tres teoremas de congruencia para triángulos — que son la base para la prueba de la relación de congruencia de figuras planas - se basan en la operación de superposición, la definición $\boldsymbol{D}_{\mathrm{XI}-10}$ elimina esta operación en el espacio y busca deducir la igualdad de los poliedros a partir de la congruencia de las figuras planas que lo forman.

Por ello resulta interesante observar que una consecuencia de la demostración de esta proposición-definición, demostración requerida por Simson y Legendre, parece ser precisamente reconocer que ésta cubre los dos casos que pueden existir: el de las figuras congruentes y el de las simétricas. ${ }^{31}$

La demostración de esta proposición-definición dada por Cauchy en 1812 consiste precisamente en hacer ver que cuando dos poliedros tienen el mismo número de caras y cada una de las caras de uno de ellos es igual (congruente) con la cara correspondiente del segundo, los dos poliedros son o bien congruentes o bien simétricos. El argumento de Cauchy consiste en mostrar que en un poliedro convexo no podrán variar los ángulos sobre sus aristas de manera que a partir de esta variación sea posible obtener un nuevo poliedro con las mismas caras, pero que no

${ }^{31}$ A pesar del "reto" que representaba esta ausencia, Legendre no pudo dar ninguna prueba de la definición euclidiana $\boldsymbol{D}_{\mathrm{XI}-10}$ sino hasta la edición 12 de sus Éléments (1823). La nota XII publicada entonces contiene la prueba mencionada, pero no es sino una versión propia de la prueba dada por Cauchy (1812), de la cual Legendre se reclama inspirador. 
sea igual al primero. El enunciado del resultado probado por Cauchy reza así:

$\boldsymbol{P}_{6}$ : En un poliedro convexo cuyas caras son invariables, los ángulos formados en las aristas que unen a dos caras, o bien la inclinación entre estas caras a lo largo de las aristas, son también invariables, de modo que con las mismas caras sólo será posible construir un poliedro que sea simétrico al primero. ${ }^{32}$

La prueba de Cauchy se basa en un resultado de la geometría plana y esférica que asegura que si en un polígono rectilíneo o esférico convexo (de más de tres lados) la longitud de los lados permanece invariable pero los ángulos formados por dichos lados varían, entonces esta variación no puede ser del mismo signo para todos ellos; lo que significa que si algunos ángulos aumentan, otros necesariamente disminuyen. Este resultado se acompaña de otro que, en este caso, asegura que el número de cambios de signo en la variación de los ángulos —el número de veces que se pasa de un ángulo cuyo valor aumenta a uno cuyo valor disminuye o viceversa - es siempre par y es al menos de cuatro variaciones. Con este doble resultado, y al seguir una idea de Legendre, Cauchy identifica un ángulo sólido $\mathfrak{A}[A]_{(B C D E \ldots)}$-formado a partir de las líneas $\mathrm{L}[A B], \mathrm{L}[A C]$, $\mathrm{L}[A D], \mathrm{L}[A E] . .$. , de las cuales no hay tres que sean coplanares, y por los ángulos planos $\mathfrak{a}[B A C], \mathfrak{a}[C A D], \mathfrak{a}[D A E] \ldots$ - con el polígono esférico $\mathrm{F}[B C D E$...], cuyos vértices $B, C, D, E \ldots$ son los puntos de intersección de una esfera con centro en el punto $A$ y radio arbitrario, con las líneas $\mathrm{L}[A B], \mathrm{L}[A C], \mathrm{L}[A D]$, $\mathrm{L}[A E], \ldots$ De acuerdo con esta forma de representar al ángulo sólido $\mathfrak{A}[A]_{(B C D E \ldots)}$, los lados $\mathrm{A}[B C], \mathrm{A}[C D], \mathrm{A}[D E], \ldots$ del polígono esférico representan los ángulos planos $\mathfrak{a}[B A C], \mathfrak{a}[C A D]$, $\mathfrak{a}[D A E] \ldots$ y los ángulos $\mathfrak{a}[B C D], \mathfrak{a}[C D E], \ldots$ representan a los

\footnotetext{
${ }^{32}$ Recherches sur les Polygones et les Polyèdres (séconde mémoire), teorema XIII.
} 
ángulos $\mathfrak{a}_{A C}, \mathfrak{a}_{A D}, \ldots$ que son los ángulos en las aristas $\mathrm{L}[A C]$, $\mathrm{L}[A D], \ldots$ (Figura 4$).{ }^{33}$

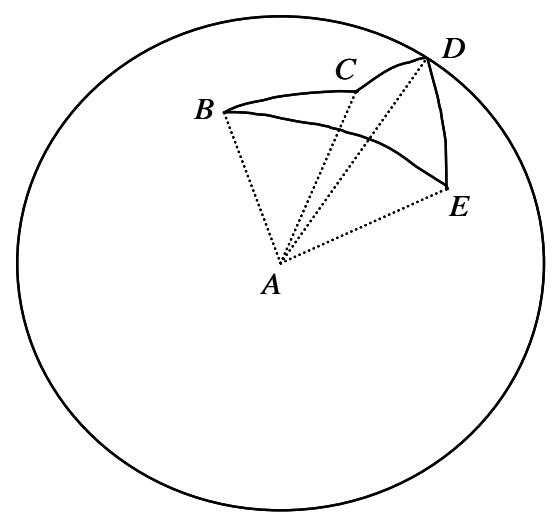

Figura 4

En las condiciones de la proposición $\boldsymbol{P}_{6}$ se asume que en un poliedro $\mathrm{K}$ sus caras permanecen invariables, y sólo se considera la posibilidad de que varíen los ángulos en las aristas, eso significa que en cada ángulo sólido del mismo sólo podrán variar los ángulos en las aristas, puesto que los ángulos planos no son sino los ángulos de las caras planas del poliedro. Los resultados sobre la geometría plana y esférica mencionados anteriormente sirven como base para concluir que en cada uno de los ángulos sólidos de un poliedro los cambios de signo en las variaciones de los ángulos en las aristas deben ser al menos cuatro. Si V representa el número de vértices del poliedro, entonces el número total de

${ }^{33}$ Esta forma de representar los ángulos sólidos permite deducir fácilmente la proposición $\boldsymbol{A}_{1}$ a partir del segundo teorema de congruencia para triángulos (la proposición I-8 del primer libro de los Elementos) y también permite ver que esta proposición no admite generalización: dos ángulos sólidos $\mathfrak{A}[A]_{(B C D E F \ldots)}$ y $\mathfrak{A}\left[A^{\prime}\right]_{\left(B^{\prime} C^{\prime} D^{\prime} E^{\prime} F^{\prime} \ldots\right)}$ pueden estar formados por el mismo número de ángulos planos — al menos cuatro - y cada ángulo plano en uno de ellos es congruente con el correspondiente ángulo plano en el otro, $\mathfrak{a}[B A C]=\mathfrak{a}\left[B^{\prime} A^{\prime} C^{\prime}\right], \mathfrak{a}[C A D]=\mathfrak{a}\left[C^{\prime} A^{\prime} D^{\prime}\right], \mathfrak{a}[D A E]=\mathfrak{a}\left[D^{\prime} A^{\prime} E^{\prime}\right], \mathfrak{a}[E A F]=$ $\mathfrak{a}\left[E^{\prime} A^{\prime} F^{\prime}\right] \ldots$ pero de esta condición no es posible deducir la igualdad de los ángulos en las aristas. 
variaciones en los ángulos de las aristas debe ser al menos de $4 \mathrm{~V}$. Sin embargo, a partir de la fórmula demostrada por Euler, que asegura que en cualquier poliedro el número de vértices, el número de caras y el número de aristas siempre están en una relación constante, Cauchy hace ver que en cualquier poliedro el número de variaciones nunca podrá ser mayor que $4 \mathrm{~V}-8$, lo que resulta contradictorio con el resultado esperado. ${ }^{34} \mathrm{La}$ conclusión de Cauchy es que en un poliedro cuyas caras permanecen invariantes, tampoco podrá haber variación en los ángulos de las aristas, con lo cual se concluye la prueba de $\boldsymbol{P}_{6}$. La única posibilidad para obtener un poliedro que teniendo las mismas caras no sea congruente con éste, es que se trate de un poliedro simétrico. A partir de ello Cauchy puede concluir, a manera de corolario, la afirmación siguiente:

$\boldsymbol{P}_{7}$ : Dos poliedros convexos, comprendidos entre el mismo número de caras iguales y colocadas de modo semejante, se pueden superponer uno sobre el otro o bien son simétricos. En ambos casos son necesariamente iguales. ${ }^{35}$

Si bien a partir de este argumento de Cauchy la definición $\boldsymbol{D}_{\mathrm{XI}-10}$ de Euclides queda plenamente demostrada, está claro que a través de $\boldsymbol{P}_{6}$ y su corolario $\boldsymbol{P}_{7}$ se reconoce la relación de simetría entre dos figuras como una modalidad de igualdad entre ellas.

Si intentamos distinguir el significado profundo de la relación que pueden guardar entre sí dos figuras sólidas que sean congruentes o bien simétricas, veremos que la única distinción que entre ellas se ha logrado establecer se deduce de la posibilidad o imposibilidad de superponer una sobre la otra. De hecho, si consideramos que la coincidencia local entre dos figuras congruentes - la congruencia entre sus caras y los ángulos que

\footnotetext{
${ }^{34}$ La fórmula de Euler, publicada en 1758 ( $f f r$. la nota 3), asegura que si $\mathrm{V}$ es el número de vértices del poliedro, $\mathrm{A}$ es el número de $\operatorname{aristas}$ y $\mathrm{C}$ el número de caras, entonces $\mathrm{V}+\mathrm{C}-\mathrm{A}=2$.

${ }^{35}$ Recherches sur les Polygones et les Polyèdres (séconde mémoire), Corolario I.
} 
ellas forman - sólo se puede constatar a partir de los teoremas de congruencia de la geometría plana, los cuales se apoyan precisamente en este procedimiento de superposición, podremos concluir entonces que es precisamente esta operación la que permite establecer el grado de coincidencia y de diferencia entre ellas.

Ya hemos hablado de las dificultades inherentes a esta operación geométrica que inaugura un nuevo estilo de prueba; la idea de aplicar una figura sobre otra no tiene justificación alguna en el marco teórico del texto euclidiano y su presencia, si bien resulta imprescindible, no admite otro fundamento que la imagen que se construye en la intuición. Como lo señalé anteriormente, el texto de los Elementos había limitado cuidadosamente la necesidad de recurrir a esta operación más allá de la geometría del plano; no obstante, en sus Éléments de Géométrie Legendre muestra la necesidad de extenderla a la geometría del espacio como el único medio a través del cual se puede dar cuenta de la relación de simetría en la geometría.

Si recordamos brevemente el modo en el que esta operación se introduce en la geometría plana euclidiana, nos encontramos con que desde un inicio su presencia es tanto más conflictiva cuanto más imprescindible resulta. En el marco teórico delineado por el sistema de postulados y Nociones Comunes, la idea de un "desplazamiento" de figuras no tiene justificación alguna en la geometría plana, por ello el sentido que puede tener la idea de que una figura sea aplicada o trasladada a un punto es aquel que consiste en justificar, a partir de estos postulados, la construcción, en el punto deseado, de una figura que sea igual a la figura dada. Sin embargo, este proyecto constructivo se agota rápidamente pues sólo culmina con éxito en un caso: cuando se trata de aplicar una línea recta sobre otra; en este caso el procedimiento constructivo ha sido justificado con las primeras tres proposiciones del libro I. Es evidente que ya en la cuarta proposición Euclides no puede ofrecer el procedimiento constructivo explícito para poder dar un contenido claro y justificado a esta operación cuando en su demostración se habla de aplicar un triángulo sobre otro. Resulta sorprendente que una vez jus- 
tificada la posibilidad de construir una línea sobre otra, el autor euclidiano pida hacer la misma operación con una figura plana, el triángulo, sin el apoyo de ninguna construcción previa. La proposición I-4 asegura que dados dos triángulos, si dos lados de uno de ellos son iguales a dos lados del otro, y además el ángulo formado por ellos es también igual al que en el segundo triángulo forman los lados correspondientes, entonces los triángulos serán iguales (congruentes). En este caso, el método demostrativo usado por Euclides consta de dos momentos: una construcción $(\kappa \alpha \tau \alpha \sigma \kappa \varepsilon v \eta)$ que consiste simplemente en asumir que uno de los triángulos puede ser aplicado ( $\dot{\epsilon} \varphi \alpha \rho \mu \delta \zeta \zeta \varepsilon \sigma \theta \alpha \iota)$ sobre el otro, y una prueba ( $\alpha \pi \delta \delta \varepsilon \iota \xi \iota \varsigma)$ en la que muestra que en esta operación los dos triángulos coinciden, lo que los hace iguales según NC.7. Pero lo relevante en este caso es que no sólo se trata de una operación que debe realizarse sin apego a ninguna cláusula constructiva explícita, sino que además el intento de proponer un proceso para la construcción de un triángulo sobre el otro parece generar necesariamente un argumento circular. En efecto, si se intenta realizar esta operación construyendo las tres líneas de un triángulo sobre las respectivas líneas del segundo, queda aún el problema de probar que al realizar esta operación las magnitudes de los ángulos que forman entre sí las líneas han sido respetadas. Este problema sólo se puede resolver a partir de la construcción, ya mencionada anteriormente, de un ángulo que sea igual a un ángulo dado. Si con base en las primeras tres proposiciones - básicamente la proposición I-3- es posible aplicar una línea sobre otra, con base en la proposición I-23 será posible ahora aplicar un ángulo sobre otro. Es posible decir que al conjuntar estos dos resultados se pueden llevar las líneas y los ángulos de uno de los triángulos sobre las líneas y los ángulos del otro, y que este procedimiento es el que globalmente se llamará la aplicación de un triángulo sobre el otro. Eso exigiría que la demostración de la proposición I-4 se pospusiera hasta que la proposición I-23 hubiese sido probada, pero la prueba de esta última supone el resultado enunciado por I-4.

$\mathrm{Al}$ señalar esta circularidad en el argumento euclidiano quiero subrayar que, a partir de esta proposición, la geometría del 
plano depende de la operación de superposición, operación no justificada constructivamente y cuyo fundamento deviene así un fundamento para la geometría misma. $\mathrm{Si}$, como hemos insistido, se trata de una operación que, expresada en términos kantianos, resulta evidente y que como tal no puede ser deducida discursivamente, aparece con claridad el carácter irreductible que la intuición desempeña en la constitución de la geometría plana como ciencia.

En el caso de la geometría del espacio tampoco existe ningún fundamento axiomático que justifique la aplicación de un cuerpo sólido sobre otro; ya hemos señalado que en los Elementos la definición $\boldsymbol{D}_{\mathrm{XI}-10}$ desempeña un papel alternativo, justamente porque pretende eliminar la necesidad de recurrir a esta operación. Parece claro que en esta perspectiva la exigencia de Simson y Legendre de una prueba para esta proposición no es otra cosa que la exigencia de corroborar si enuncia de otra manera lo que la operación de superposición, justificada o no constructivamente, permite ver.

Para el caso de la proposición $\boldsymbol{A}_{1}$, Legendre hace ver, como también lo hizo Simson, que el recurso a esta operación de superposición es necesario, no sólo porque sobre ella se apoya la demostración de que en un ángulo sólido triedro la igualdad de los ángulos en las aristas se deduce de la igualdad de los ángulos planos, sino porque en las condiciones de esta proposición sólo ella permite distinguir si se trata de dos ángulos sólidos que son congruentes o bien si son simétricos. Igualmente, en la proposición XI-26 de los Elementos esta operación de superposición es necesaria para distinguir si el ángulo sólido triedro construido es congruente con el ángulo sólido dado o simétrico a él. Por ello no creo posible suponer que esta operación sea la manifestación intuitiva de una construcción efectivamente realizada; más bien parece que la prueba de que la construcción realizada es la construcción requerida se justifica a través de la operación de superposición.

Está claro que en la prueba de Cauchy para la definición $\boldsymbol{D}_{\mathrm{XI}-10}$ la operación de superposición está totalmente ausente; sin embargo, no lo está de las conclusiones que de esta prueba 
se pueden extraer. Para Cauchy, el enunciado $\boldsymbol{D}_{\mathrm{XI}-10}$ es equivalente a la proposición $\boldsymbol{P}_{6}$, a pesar de que en el primero sólo se menciona la relación de igualdad como caso posible conforme a la hipótesis de la igualdad de las caras de los poliedros, mientras que en la segunda se consideran dos casos que la igualdad de las caras de los poliedros no permite distinguir.

De acuerdo con NC.7, la igualdad de las figuras y de las magnitudes se deduce de su coincidencia. Esta afirmación constituye, en palabras de Kant (Prolegómenos), una proposición sintética que se apoya en la intuición inmediata, que, dada la ausencia de la construcción necesaria para justificarla, es el único soporte de la operación requerida. Pero la conclusión de que dos figuras son iguales por simetría es también una proposición sintética que se apoya en la intuición inmediata.

Desde la primera edición de sus Grundlagen der Geometrie, Hilbert insistió en la doble necesidad de probar tanto la independencia relativa y la consistencia de los axiomas de la geometría plana como el carácter distinto al que ellos se refieren. Los axiomas de la geometría plana expresan relaciones de incidencia, relaciones de orden y relaciones de congruencia, y la independencia entre ellos es una consecuencia del hecho de que se trata de relaciones que son distintas e irreductibles unas a otras. A la luz de esta distinción resulta claro el problema al que nos enfrentan $N C .7$ y $N C .8$ : ambas aluden a una relación de igualdad o desigualdad entre magnitudes geométricas, pero evidentemente esta relación se refiere al "tamaño" de las magnitudes - lo que compete a una relación de congruencia en la terminología de Hilbert. El problema con estas dos Nociones Comunes es que el criterio sobre el cual se apoyan tiene que ver con la coincidencia plena o parcial de los objetos geométricos que representan a dichas magnitudes; vale decir que la relación entre estas magnitudes se deriva del modo en que estos objetos inciden, ya sea de manera plena o sólo parcial, uno sobre otro.

A la luz de esta observación de Hilbert, hay otro aspecto ya señalado por Kant en su primera aproximación a la relación de simetría que cobra particular relevancia. Nos referimos a su observación de la posibilidad de comprender este problema de 
la simetría a partir de la teoría leibniciana del Analysis situs. De acuerdo con lo que Legendre establece en sus definiciones, la diferencia esencial entre dos sólidos simétricos radica no en las magnitudes planas que los constituyen sino en la disposición que ellas guardan entre sí, en la orientación o, si se quiere, en la situación en la que se encuentran.

Si recordamos el ejemplo favorito de Kant, la relación de la mano derecha con la mano izquierda, tal y como es analizado en el texto Regiones, la prueba de la existencia de un espacio absoluto se deriva del hecho de que en un espacio puramente relacional no podrían existir simultáneamente ambas manos; pero hay en este texto un argumento ofrecido por Kant que permite ver que no sólo la existencia de una de las dos manos acepta la existencia de la otra, sino que es en su relación con ese espacio absoluto en el que ambas se encuentran donde se puede dar cuenta de aquello que las hace diferentes:

Si nos hacemos a la idea de que el primer objeto de la creación es una mano humana, es necesario que sea una mano derecha o una mano izquierda; y para producir una de ellas es necesaria una acción de la causa creadora que sea distinta de aquella por la que se podría producir a su contraparte. ${ }^{36}$

Esta imagen que algunos comentadores califican como un experimento mental ${ }^{37}$ corresponde, a nuestro juicio, con la construcción euclidiana del ángulo sólido triedro que presentamos anteriormente: dado un ángulo sólido $\mathfrak{A}[A]_{(B C D)}$ formado por los ángulos planos $\mathfrak{a}[B A C], \mathfrak{a}[C A D]$ y $\mathfrak{a}[B A D]$, la geometría del espacio dispone tan sólo de la posibilidad de construir el ángulo sólido $\mathfrak{A}\left[A^{\prime}\right]_{\left(B^{\prime} C^{\prime} D^{\prime}\right)}$ y el ángulo sólido $\mathfrak{A}\left[A^{\prime}\right]_{\left(B^{\prime} C^{\prime \prime} D^{\prime \prime}\right)}$; la diferencia en la "acción creadora" radica, como se hizo ver, en la elección del lado de la línea $\mathrm{L}\left[A^{\prime} B^{\prime}\right]$ en el que se construyan los ángulos $\mathfrak{a}\left[B^{\prime} A^{\prime} C^{\prime}\right]$ y $\mathfrak{a}\left[B^{\prime} A^{\prime} E^{\prime}\right]$, o $\mathfrak{a}\left[B^{\prime} A^{\prime} C^{\prime \prime}\right]$ y $\mathfrak{a}\left[B^{\prime} A^{\prime} E^{\prime \prime}\right]$ que son iguales respectivamente a los ángulos $\mathfrak{a}[B A C]$ y $\mathfrak{a}[B A E]$. A

${ }^{36}$ Regiones, sección IV.

${ }^{37}$ Por ejemplo, William Harper, "Kant on Incongruent Counterparts", en J. van Cleve y R. Frederick (comps.), The Philosophy of Right and Left. 
partir de esta diferencia, es distinta la orientación de los ángulos planos $\mathfrak{D}\left[\mathfrak{a}\left[B^{\prime} A^{\prime} C^{\prime}\right], \mathfrak{a}\left[B^{\prime} A^{\prime} D^{\prime}\right], \mathfrak{a}\left[C^{\prime} A^{\prime} D^{\prime}\right]\right]$ y $\mathfrak{D}\left[\mathfrak{a}\left[B^{\prime} A^{\prime} C^{\prime \prime}\right]\right.$, $\left.\mathfrak{a}\left[C^{\prime \prime} A^{\prime} D^{\prime \prime}\right], \mathfrak{a}\left[B^{\prime} A^{\prime} D^{\prime \prime}\right]\right]$, y una de ellas es necesariamente la misma que la orientación de los ángulos planos del ángulo sólido $\mathfrak{A}[A]_{(B C D)}$. Pero si a partir de esta diferencia en la orientación de los ángulos planos hemos hecho ver la posibilidad o la imposibilidad de la congruencia a través de la operación de superposición, ello depende de que en el espacio (de dos dimensiones en este caso) en el que esta construcción se haga, pueda hablarse de que para una línea $\mathrm{L}\left[A^{\prime} B^{\prime}\right]$ existen efectivamente dos lados. Sabemos que esta condición no es otra que la de la orientabilidad del espacio - condición asumida de manera automática por toda la geometría desde Euclides hasta Legendre - y que Kant consideró primero como una condición inherente al espacio absoluto, para concebirla más tarde como una capacidad del sujeto para distinguir una orientación en el orden en el que se presentan las figuras, capacidad que no radica en la razón sino que es una condición fundamental de nuestra intuición espacial.

De este modo podemos concluir que, sin pretender minimizar las diferencias que existen entre los distintos momentos en los que Kant se ocupa de las parejas incongruentes, en los tres textos a los que he hecho referencia, sus observaciones resultan clave para comprender la naturaleza de la relación de simetría. En las Regiones, Kant subraya que para entender esta relación es necesario considerar la posición de las partes componentes de los cuerpos, posición que él refiere a un espacio real y absoluto - aun cuando ya hemos mostrado que la condición que se pide del espacio es que sea un espacio orientable. En la Dissertatio, Kant subraya el hecho de que el conocimiento geométrico encuentra su fundamento en una intuición pura, la del espacio, y que sobre ella puede darse un fundamento para la comprensión de la relación de simetría. Finalmente, en los Prolegómenos Kant ahonda en este carácter del conocimiento geométrico basado en la intuición pura del espacio, pero permite ahora pensar, lo que parece ser la lección última de la prueba de Cauchy de 1812, que la relación de simetría no es sino otro modo de concebir la relación de igualdad. 
Podremos concluir así que si el único criterio del que dispone la geometría euclidiana para concluir si dos figuras son congruentes o simétricas es su coincidencia, o la falta de ella cuando una se aplica sobre la otra - lo que en cualquier caso nos lleva a establecer una proposición sintética basada en la intuición inmediata - la posibilidad de determinar cuál de las dos relaciones tiene lugar entre las dos figuras resulta trivial si se dispone legítimamente de esta operación, e imposible si se carece de ella.

\section{BIBLIOGRAFÍA}

Álvarez, C., 2003, “Two Ways of Reasoning and Two Ways of Arguing in Geometry. Some Remarks Concerning the Application of Figures in Euclidean Geometry", Synthese, no. 134, pp. 289-323.

Cauchy, A.L., 1812, Recherches sur les Polygones et les Polyèdres (séconde mémoire), Journal de l'École Polytechnique, vol. 16, cuaderno 9, París.

Euclides, 1883-1899, Elementa, L. Heiberg y H. Menge (comps.), Teubner, Leipzig. [Versión en castellano: Elementos, trad. y comentarios M. Puertas, Gredos, Madrid, 1991; versión en inglés: The Thirteen Books of the Elements, trad. y comentarios Th. Heath, Cambridge University Press, Nueva York [1908] 1956.]

Euler, L., 1758, Elementa doctrince solidorum. Demonstratio nonnullarum insignium proprietatum, quibus solida hedris planis inclusa sunt praedita, Novi comment. Acad. Sc. Imp. Petropol [4], Opere Omnia, vol. 1, no. 26 [1752-1753], pp. 109-160.

—_ 1750, Reflexions sur l'espace et le temps, Mémoires de l'Academie des Sciences de Berlin [4], Opere Omnia, vol. 3, no. 2 [1748], pp. 324-333.

Hilbert, D., 1903, Grundlagen der Geometrie, 2a. ed., Teubner, Leipzig. [Versión en inglés: Foundations of Geometry, Open Court, Chicago, 1971.]

Kant, I., 1783, Prolegomena zu einer jeden künftigen Metaphysik die als Wissenschaft wird auftreten können. [Versión en castellano: Prolegómenos a toda metafísica futura, trad. Julián Besteiro, Aguilar, Buenos Aires, 1975; versión en inglés: Prolegomena to Any Future Metaphysics, Bobbs-Merrill, Indianápolis, 1950; versión en francés: Prolégomènes à Toute Métaphysique Future, Vrin, París, 2001.] 
Kant, I., 1770, De Mundi Sensibilis atque Intelligibilis Forma et Principiis, Dissertatio. [Versión en inglés: "Dissertation on the Form and Principles of the Sensible and Intelligible World", en Kant's Inaugural Dissertation and Early Writings on Space, Open Court, Chicago, 1929; versión en francés: Dissertation sur la Forme et les Principes du Monde Sensible et du Monde Intelligible, Vrin, París, 1995.]

- - 1768, Von dem ersten Grande des Unterschiedes der Gegenden im Raume. [Versión en castellano: "Del primer fundamento de la diferencia de las regiones del espacio", en Opúsculos de filosofía natural, trad. Alianza Editorial, Madrid, 1992; versión en inglés: On the First Ground of the Distinction of Regions in Space, en Kant's Inaugural Dissertation and Early Writings on Space, Open Court, Chicago, 1929.]

_- 1763, Versuch, den Begriff der negativen Grössen in die Weltweisheit einzuführen. [Versión en castellano: "Ensayo para introducir las magnitudes negativas en filosofía", en Opúsculos de filosofía natural, Alianza Editorial, Madrid, 1992.] [Versión en francés: Essai pour Introduire en Philosophie le Concept de Grandeur Négative, Vrin, París, 1980.]

Legendre, A.M., 1823, Éléments de Géométrie, 12a. ed., Firmin Didot, París.

Simson, R., 1756, The Elements of Euclid, A. y J. Duncan, Glasgow. Van Cleve, J., y R. Frederick (comps.), 1991, The Philosophy of Right and Left, Kluwer Academic Publisher, Dordrecht.

Vleeschauwer, J.H. de, 1797 (1934), La Déduction trascendentale dans l'œuvre de Kant, París.

Recibido el 24 de enero de 2003; revisado el 20 de octubre de 2003; aceptado el 5 de noviembre de 2003. 